\title{
Chemical Composition of the Galactic Bulge in Baade's Window
}

\author{
Andrew McWilliam ${ }^{1}$, Jon Fulbright ${ }^{2}$, R. Michael Rich ${ }^{3}$ \\ ${ }^{1}$ Carnegie Observatories, 813 Santa Barbara Street, Pasadena, CA, USA \\ email: andy@obs.carnegiescience.edu \\ ${ }^{2}$ Johns Hopkins University, Physics \& Astronomy, N. 3400 Charles St., Baltimore, MD, USA \\ ${ }^{3}$ University of California, Los Angeles, Physics \& Astronomy, 430 Portola Plaza, Box 951547, \\ Los Angeles, CA, USA
}

\begin{abstract}
McWilliam and Zoccali (2009) show the existence of two Red Clump populations towards the Galactic bulge, based on 2MASS data. 2.Measured $[\mathrm{Mg} / \mathrm{Fe}],[\mathrm{Al} / \mathrm{Fe}]$, and $[\mathrm{La} / \mathrm{Eu}]$ ratios in the bulge are consistent with a rapid formation timescale $(<1 \mathrm{Gyr})$, which also requires a slightly top-heavy IMF to reproduce the mean bulge metallicity. The $[\mathrm{C} / \mathrm{O}]$ and $[\mathrm{O} / \mathrm{Fe}]$ ratios are consistent if their predicted metal-dependent yields from massive stars with winds are considered. The decline in explosive $[\alpha / \mathrm{Fe}](\mathrm{Si}, \mathrm{Ca}$, and $\mathrm{Ti}$ ) can only be understood if their yields also decline with metallicity above $[\mathrm{Fe} / \mathrm{H}] \sim-1$.
\end{abstract}

Keywords. stars: abundances, Galaxy: abundances, Galaxy: bulge, nucleosynthesis.

\section{First, Something Completely Different...}

From 2MASS data McWilliam \& Zoccali (2009, in preparation) show the presence of two red clumps towards the Galactic bulge. These red clumps are approximately at a distance of 6 and $8 \mathrm{Kpc}$ and cover an area approximately $20 \times 20$ degrees on the sky. These structures are reminiscent of the X-shaped or peanut-shaped features in the predictions of Patsis, Skokos, \& Athanassoula (2002).

\section{Introduction}

Detailed abundance ratios can constrain the bulge formation timescale, SFR, IMF as well as the role of accretion in bulge history, and stellar nucleosynthesis yields. Enhanced $\alpha$-element abundances $(\mathrm{O}, \mathrm{Mg}, \mathrm{Si}, \mathrm{Ca}, \mathrm{Ti})$, relative to $\mathrm{Fe}$, in the Galactic halo (e.g. Wallerstein 1962; Conti et al. 1967) are thought to arise from the time delay between Fe produced by Type II and Type Ia supernovae (henceforth SNII and SNIa); see Tinsley (1979). Matteucci \& Brocato (1990) showed that the star formation rate (SFR) determines the $[\mathrm{Fe} / \mathrm{H}]$ where the $[\alpha / \mathrm{Fe}]$ ratio declines (the knee) due to the late addition of SNIa iron. Smecker-Hane \& Wyse (1992) found a mean SNIa timescale of 1Gyr. Because bulges are thought to have formation timescales shorter than SNIa progenitor lifetimes they should possess enhanced $[\alpha / \mathrm{Fe}]$ even at solar metallicity. Wyse \& Gilmore (1993) showed that a top heavy massive star initial mass function (IMF) leads to larger mean enhancement of $[\mathrm{O} / \mathrm{Fe}]$ before the knee. This work is motivated by a simple question: What was the formation timescale of the Galactic bulge? 

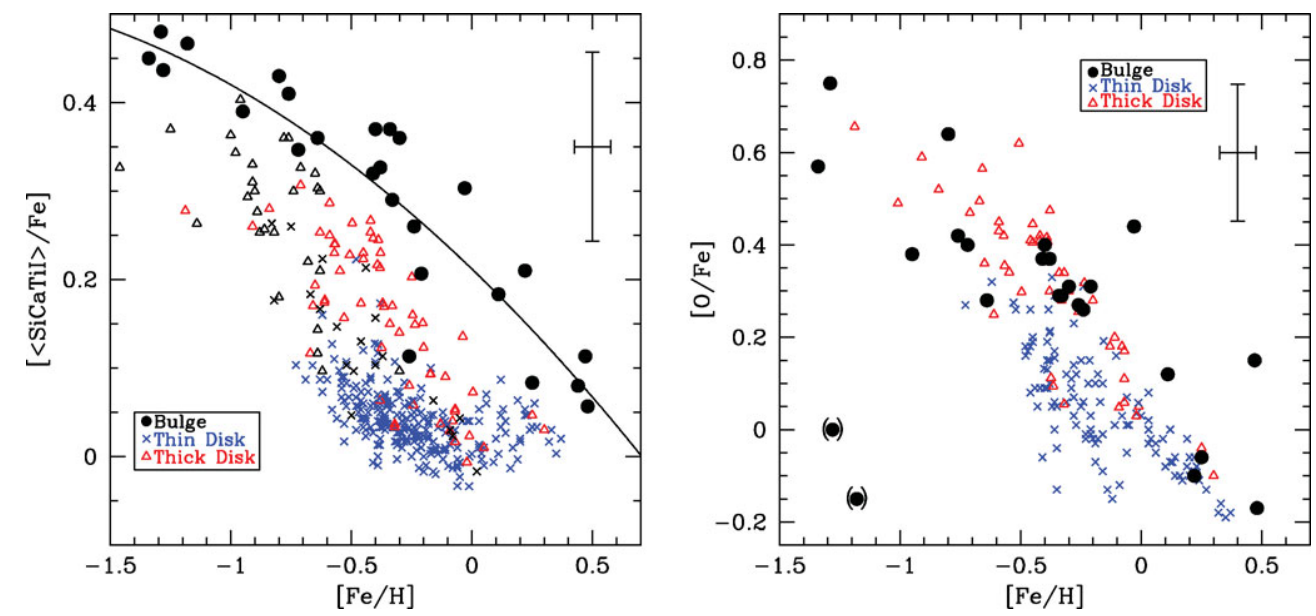

Figure 1. a)Left: Explosive alpha-elements in the bulge and disk (see FMR07) b)Right: Revised $[\mathrm{O} / \mathrm{Fe}]$ in the bulge and disk (see McWilliam, Fulbright \& Rich 2009, in preparation)

\section{The Mean Metallicity-Age Problem}

The mean of the bulge metallicity distribution function is near the solar value (e.g. Zoccali et al. 2008). The old age, from main sequence turnoffs, and short dynamical timescale suggest that the bulge formed rapidly; this is supported by the enhanced $[\mathrm{Mg} / \mathrm{Fe}] \mathrm{ra}-$ tios near solar metallicity (e.g. McWilliam \& Rich 1994; Lecureur et al. 2007; Fulbright, McWilliam \& Rich 2007, henceforth FMR07). Chemical evolution model fits to these abundances, by Ballero et al. (2007), gives a bulge formation timescale of $\sim 0.7$ Gyr.

The combination of rapid formation and mean solar metallicity presents a problem. In a rapid formation no significant Fe is contributed by SNIa, so the mean yield of metals is lower than normal. In a rapidly formed bulge the mean metallicity should be lower than the solar neighborhood, by more than $\sim 0.3$ dex. To fit the observed solar metallicity a rapidly formed bulge must also have had a higher effective mean yield of metals; Ballero et al. (2007) achieved this using a top-heavy IMF.

\section{Alpha-Elements in the Galactic Bulge}

\subsection{Explosive Alphas}

By ratioing the measured $\alpha$-element abundances for a sample of Galactic bulge RGB stars FMR07 established that the trends of $\mathrm{Ca}, \mathrm{Si}$ and $\mathrm{Ti}$ were indistinguishable, while $\mathrm{Mg}$ and $\mathrm{O}$ had unique trends $(\mathrm{Mg}$ increased while $\mathrm{O}$ decreased with metallicity relative to $\mathrm{Ca}$, $\mathrm{Si}$ and $\mathrm{Ti}$ ). Given the common trends for $\mathrm{Si}, \mathrm{Ca}$ and $\mathrm{Ti}$, and because these elements are thought to be produced during explosive nucleosynthesis (e.g., Woosley \& Weaver 1995) FMR07 employed the average explosive $\alpha$ abundance from these three elements. Figure 1a shows the average $[<\mathrm{Si}, \mathrm{Ca}, \mathrm{TiI}>/ \mathrm{Fe}]$ ratios of the FMR07 RGB sample compared to the thick and thin Galactic disk dwarf star results. In Figure 1a the bulge $[<\mathrm{SiCaTi}>/ \mathrm{Fe}]$ ratio declines by $\sim 0.4$ dex, with small scatter, from the most metal-poor to the most metal-rich stars and is higher than the thick and thin disk trends.

The FMR07 results for Si, Ca and Ti, interpreted in the Tinsley (1979) paradigm, indicates that a considerable amount of iron from SNIa has reduced the $[\alpha / \mathrm{Fe}]$ ratio over the range of metallicities covered by the bulge. This downward trend and the low-metallicity onset of the knee suggests that bulge evolution occurred over a long timescale greater than $1 \mathrm{Gyr}$, perhaps significantly longer. On the other hand, the $\sim 0.2$ dex enhancement of $[<$ SiCaTiI $>/ \mathrm{Fe}]$ at solar metallicity might indicate a shorter evolution timescale than 

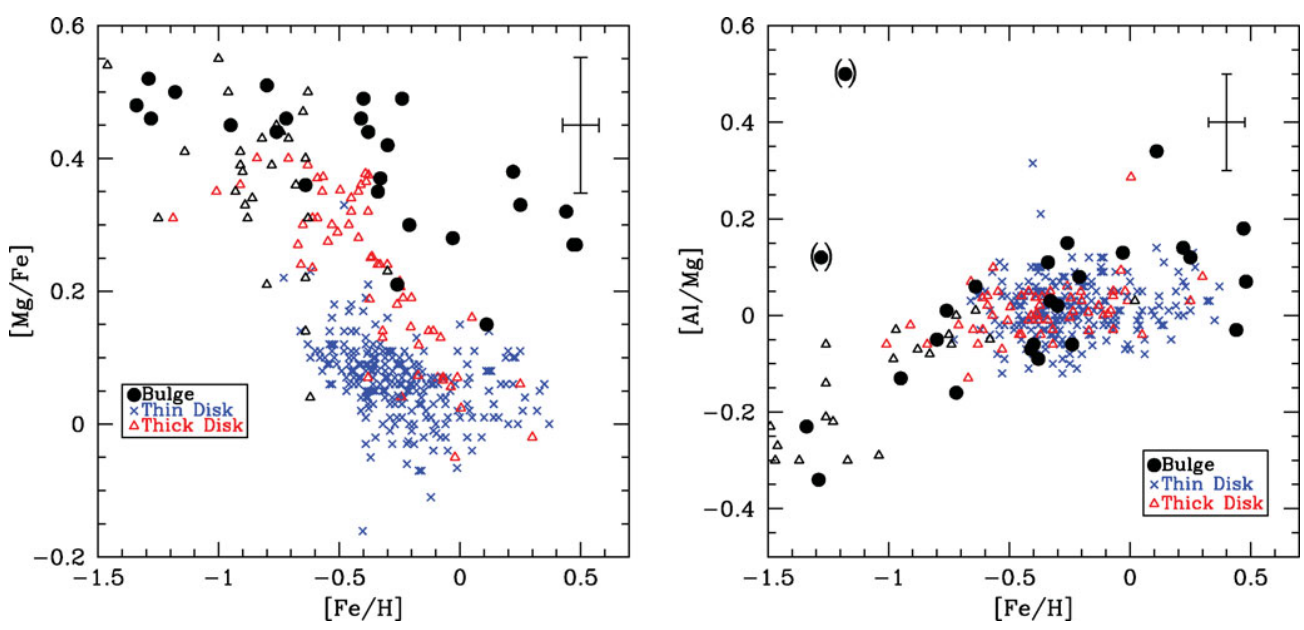

Figure 2. a) Left: $\quad[\mathrm{Mg} / \mathrm{Fe}]$ in the bulge and disk (from FMR07) b)Right: $\quad[\mathrm{Al} / \mathrm{Mg}]$ in the bulge and disk (from FMR07)

for the Galactic thin disk, and the overall higher $[\alpha / \mathrm{Fe}]$ values may be understood with an IMF slightly more top-heavy than for the thin disk.

\subsection{Hydrostatic Alphas}

The original FMR07 bulge star $[\mathrm{O} / \mathrm{Fe}]$ trend was slightly enhanced relative to the solar neighborhood, but declined by $\sim 0.7$ dex from $[\mathrm{Fe} / \mathrm{H}]=-1.5$ and +0.5 dex. FMR07 employed $\mathrm{Fe}$ II lines in the ratio for robust $\mathrm{O} / \mathrm{Fe}$ ratio measurements; however, the $\mathrm{C}$ abundance required to account for $\mathrm{CO}$ formation was adopted assuming the solar $\mathrm{C} / \mathrm{Fe}$ ratio, adjusted for RGB dredge-up. The FMR07 oxygen abundances were recently revised by McWilliam, Fulbright and Rich (2009, in preparation, MFR09). In this case we employed the $\mathrm{C} / \mathrm{Fe}$ abundances determined from the near-infrared $\mathrm{Fe}$ and $\mathrm{CO}$ molecular lines by Meléndez et al. (2008). Because well over $90 \%$ of the atmospheric carbon is in the form of $\mathrm{CO}$ in RGB stars, total carbon abundances derived from CO lines are more reliable than from other species. Figure $1 \mathrm{~b}$ shows the new $[\mathrm{O} / \mathrm{Fe}]$ trend for Galactic bulge stars from MFR09, which appears very similar to the trend seen in the thick disk; certainly, there is no compelling evidence that the trend differs significantly from the thick disk. This conclusion supports that of Meléndez et al. (2008), who employed OH lines, rather than the $[\mathrm{O}$ I] forbidden line at $6300 \AA$ used by FMR07 and MFR09.

Figure $1 \mathrm{~b}$ indicates a steeper decline of $[\mathrm{O} / \mathrm{Fe}]$ than seen in the explosive alpha-elements of Figure 1a. In the Tinsley paradigm the decline is due to iron from SNIa, thus indicating a long formation timescale for the bulge and thick disk, greater than 1 Gyr. SNIa nucleosynthesis models (e.g. Nomoto, Thielemann, \& Yokoi 1984) indicate production of the explosive alpha elements, but an insignificant $[\mathrm{O} / \mathrm{Fe}]$ ratio, which might explain the steeper decline in $[\mathrm{O} / \mathrm{Fe}]$ than $[<\mathrm{SiCaTi}>/ \mathrm{Fe}]$ in the bulge.

The consistency of the picture presented so far is destroyed by the $\mathrm{Mg}$ and $\mathrm{Al}$ abundances in the FMR07 bulge stars. Figure $2 \mathrm{a}$ shows the $[\mathrm{Mg} / \mathrm{Fe}]$ trend in the bulge compared to thin and thick disk stars. At solar metallicity $[\mathrm{Mg} / \mathrm{Fe}]$ is $\sim 0.4$ dex; even the most metal-rich bulge stars, near $[\mathrm{Fe} / \mathrm{H}]=+0.5$, have $[\mathrm{Mg} / \mathrm{Fe}]$ ratios of $\sim 0.3$ dex. A slight decline in $[\mathrm{Mg} / \mathrm{Fe}]$ from $[\mathrm{Fe} / \mathrm{H}]=-1.5$ to +0.5 might be due to Fe from SNIa, but this is smaller than the decline in $[\mathrm{O} / \mathrm{Fe}]$ by $\sim 0.5 \mathrm{dex}$, and smaller than the decline in $[<\mathrm{SiCaTi}>/ \mathrm{Fe}]$ by $0.15 \mathrm{dex}$. It is not possible to explain the decline in $[\alpha / \mathrm{Fe}]$ of the explosive $\alpha$-elements, $\mathrm{O}$ and $\mathrm{Mg}$ with only the addition of iron from SNIa. The relatively 
small decline in $\mathrm{Mg} / \mathrm{Fe}$ is the most difficult to comprehend in light of a time-delay scenario for oxygen and the explosive $\alpha$-elements. Indeed, from a strict delay scenario the $[\mathrm{Mg} / \mathrm{Fe}]$ ratios indicate that the bulge formed very rapidly, in less than $1 \mathrm{Gyr}$, while the $[\mathrm{O} / \mathrm{Fe}]$ ratios suggest slow formation, on the timescale of the Galactic disk, significantly longer than 1 Gyr. This is particularly surprising because $\mathrm{O}$ and $\mathrm{Mg}$ are both thought to be formed in the hydrostatic phase of core-collapse SN progenitors, while neither element is predicted to have significant production from SNIa.

Because the implied formation timescale for the bulge is very dependent on the $\mathrm{Mg}$ abundances FMR07 considered additional Mg I lines, but found the same result as initially obtained. Enhanced $\mathrm{Mg}$ abundances are also supported by the results of Lecureur et al. (2007) and McWilliam \& Rich (1994, 2004). Finally, FMR07 employed Al as a proxy for Mg. Arnett (1971) noted that in massive stars the odd/even yield ratios, as indicated by $[\mathrm{Al} / \mathrm{Mg}]$, should depend on the neutron excess, i.e., proportional to $[\mathrm{Fe} / \mathrm{H}]$. Figure $2 \mathrm{~b}$ shows the FMR07 $[\mathrm{Al} / \mathrm{Mg}]$ versus $[\mathrm{Fe} / \mathrm{H}]$ for the bulge compared with the thin and thick disks; the trends appear to be identical. If FMR07 had erroneously overestimated $\mathrm{Mg}$ then the $\mathrm{Al}$ abundances must also be overestimated by the same amount, which seems unlikely. Therefore, the $\mathrm{Mg}$ and $\mathrm{Al}$ abundances are consistent and enhanced in the bulge $\mathrm{RGB}$ stars, even at $[\mathrm{Fe} / \mathrm{H}]=+0.5$. In the Tinsley time-delay scenario these $\mathrm{Mg}$ and $\mathrm{Al}$ enhancements indicate a rapid bulge formation timescale. A serious problem is that abundances of lensed bulge dwarf stars, reported by Bensby at this meeting, disagree with the $[\mathrm{Mg} / \mathrm{Fe}]$ trends found for unlensed bulge RGB stars.

McWilliam \& Rich (2004) proposed that the different bulge trends for $[\mathrm{O} / \mathrm{Fe}]$ and $[\mathrm{Mg} / \mathrm{Fe}]$ resulted from reduced oxygen yields from massive stars from stripping of their envelopes by metal-dependent winds, as expected in the formation of Wolf-Rayet stars. Bulge chemical evolution models by McWilliam et al. (2008) and Cescutti et al. (2009) using metal-dependent oxygen yields from massive stars with mass-loss, from Maeder (1992, M92) and Meynet \& Maeder (2002, MM02), and semi-empirical Mg yields, found qualitative agreement with the observed, universal, decline in $[\mathrm{O} / \mathrm{Mg}]$ versus $[\mathrm{Mg} / \mathrm{H}]$. Thus, it appears that the decline in $[\mathrm{O} / \mathrm{Mg}]$ with $[\mathrm{Mg} / \mathrm{H}]$ is consistent with metal-dependent oxygen yields from massive stars, and indicates that the decline in $[\mathrm{O} / \mathrm{Fe}]$ is not due to the addition of iron from SNIa.

If metallicity-dependent winds are responsible for reducing the oxygen yields from massive stars then the $[\mathrm{C} / \mathrm{O}]$ yield ratio should increase with metallicity. Cescutti et al. (2009) investigated chemical evolution models for [C/O] ratios using massive star $\mathrm{C}$ and O yields, compared with the revised [C/O] ratios from MFR09. The bulge chemical evolution model adopted a rapid formation timescale and top-heavy IMF, consistent with Ballero et al. (2007). The MFR09 abundance measurements showed a $\sim 0.7$ dex increase in $[\mathrm{C} / \mathrm{O}]$ with $[\mathrm{O} / \mathrm{H}]$. WW95 element yields without mass-loss failed to match the observations, while the models with mass-loss closely matched the observed trend, and bracketed the observations. It appears that a mass-loss rate higher than the MM02 but lower than M92 would best fit the [C/O] observations. One inconsistency is the metalpoor bulge stars, which show a higher [C/O] plateau than expected; Cescutti et al. (2009) suggested that the required source of carbon may be from a population of low-metallicity Wolf-Rayet stars formed via binary Roche-lobe stripping of the envelopes.

The conclusion is that the observed $[\mathrm{O} / \mathrm{Fe}],[\mathrm{C} / \mathrm{O}]$ ratios, and the $\mathrm{Mg}$ and $\mathrm{Al}$ abundances are consistent with the high SFR, short bulge formation timescale and top-heavy IMF obtained by Ballero et al. (2007). If correct, this conclusion means that the decline of the $[<\mathrm{SiCaTi}>/ \mathrm{Fe}]$ ratios in Galactic bulge stars cannot be due to increased iron production by SNIa. FMR07 proposed a solution to this puzzle: that the yields for explosive alpha elements decline with increasing metallicity in SNII. 


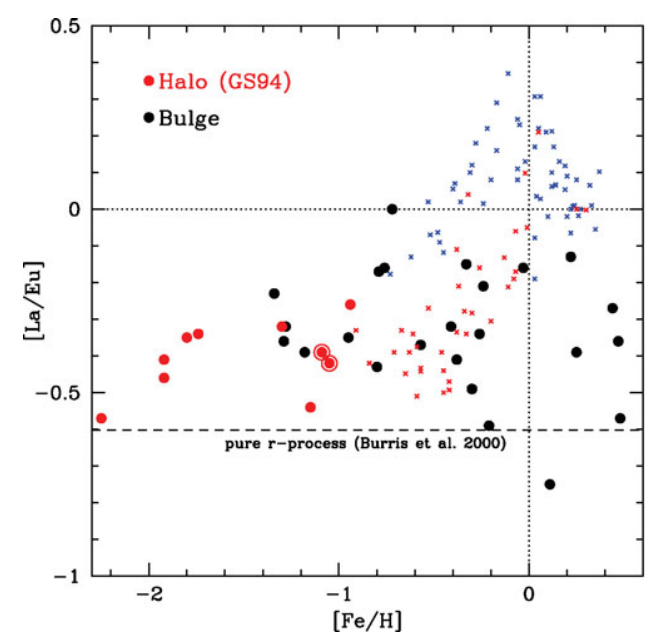

Figure 3. a)Left: $[\mathrm{La} / \mathrm{Eu}]$ in the bulge and disk Blue and red crosses: thin and thick disk stars. (see Fulbright et al. 2009, in progress)

\section{Neutron-Capture Elements}

Figure 3a compares the $[\mathrm{La} / \mathrm{Eu}]$ ratios in the halo, bulge, thick disk and thin disk. This ratio is sensitive to the relative importance of the s- and r-processes; higher values indicate a greater s-process fraction. Since the main s-process is thought to be produced by relatively low-mass AGB stars an enhanced s-process fraction (i.e., high [La/Eu]) indicates long timescales. The r-process is thought to be produced in core-collapse supernova events (i.e. massive stars on short timescales) with low $[\mathrm{La} / \mathrm{Eu}]$ ratios. Figure 3a also indicates the solar-system pure r-process $[\mathrm{La} / \mathrm{Eu}]$ ratio as determined by Burris et al. (2000). It is clear that our bulge [La/Eu] ratios (Fulbright et al. 2009, in progress) are halo-like and closer to the pure r-process value than the thin disk trend. This indicates a rapid bulge formation timescale, qualitatively consistent with our conclusions from $[\mathrm{Mg} / \mathrm{Fe}]$ and $[\mathrm{Al} / \mathrm{Mg}]$. The $[\mathrm{La} / \mathrm{Eu}]$ ratio certainly indicates that the bulge formed more rapidly than the thin disk. Much of the thick disk shares the halo-like [La/Eu] ratios, but some of the thick-disk points indicate higher s-process contributions. Therefore, either the thick disk evolved more slowly than the bulge, but faster than the thin disk, or some of the thick disk stars are mis-identified thin disk interlopers.

One difficulty with the s- to r-process ratio is that the r-process astrophysical site is not known; even the neutron source for the r-process is unconstrained. Figure 3b shows the $[\mathrm{Eu} / \mathrm{Fe}]$ in the bulge compared to the thick disk and thin disk, which measures the r-process/Fe ratio. If the r-process is made only by core-collapse $\mathrm{SNe}$, then, naively, the alpha-like decline in $[\mathrm{Eu} / \mathrm{Fe}]$ with $[\mathrm{Fe} / \mathrm{H}]$ in the disk, which follows the $[\alpha / \mathrm{Fe}]$ trend, is due to a declining SNII/SNIa ratio as in the Tinsley (1979) scenario. Therefore, Figure $3 \mathrm{~b}$ indicates that the disk-like trend of the bulge $[\mathrm{Eu} / \mathrm{Fe}]$ ratio is due to the addition of iron from SNIa ejecta, and suggests that the bulge formed on a long timescale, contrary to our conclusion from the bulge $[\mathrm{La} / \mathrm{Eu}]$ ratios.

\section{Summary and Conclusions}

A naive interpretation of the $[\alpha / \mathrm{Fe}]$ ratios in bulge RGB stars leads to contradictory conclusions about the bulge formation timescale, depending on the $\alpha$ elements employed. The explosive alphas $[<\mathrm{SiCaTi}>/ \mathrm{Fe}]$ and the hydrostatic $[\mathrm{O} / \mathrm{Fe}]$ decline strongly with $[\mathrm{Fe} / \mathrm{H}]$ above -1 and suggest contribution of SNIa iron and a slow formation ( $>1 \mathrm{Gyr})$. 
On the other hand, the $[\mathrm{Mg} / \mathrm{Fe}]$ abundance ratio trend suggests almost no Fe from SNIa, and a formation timescale $\sim 0.7 \mathrm{Gyr}$. The $\mathrm{Mg}$ abundance enhancements are corroborated by results from independent researchers, and the $[\mathrm{Al} / \mathrm{Mg}]$ ratio (Figure $2 \mathrm{~b}$ ). Chemical evolution models, including mass-loss from massive stars, with a rapid bulge formation timescale and a top-heavy IMF predict $[\mathrm{O} / \mathrm{Mg}]$ and $[\mathrm{C} / \mathrm{O}]$ trends consistent with observations; contrary to the simplistic interpretation, the oxygen abundances are consistent with the $\mathrm{Mg}$ result. This represents a departure from the original time-delay scenario suggested by Tinsley (1979). Additional abundance probes come from the neutron capture elements. The bulge $[\mathrm{La} / \mathrm{Eu}]$ ratios are halo-like even for the most metal-rich bulge stars, suggesting that the bulge evolved rapidly, similar to the halo. Surprisingly, the $[\mathrm{Eu} / \mathrm{Fe}]$ trend, which naively should follow the $\alpha$-elements, is similar to that of the solar neighborhood disk and thus supports the longer bulge formation timescale. However, since the r-process is not well understood we take the position that the $[\mathrm{Eu} / \mathrm{Fe}]$ trend tells us more about the nature of the r-process than the bulge formation timescale.

We conclude that our measured element ratios are consistent with a rapid bulge formation timescale of less than $\sim 1$ Gyr, that the bulge IMF was top-heavy, and that the explosive $[\alpha / \mathrm{Fe}]$ and $[\mathrm{O} / \mathrm{Fe}]$ trends involve metallicity dependent yields in addition to the Tinsley time-delay effect. Thus, the $\alpha$-elements are much more interesting than we originally thought.

\section{Acknowledgements}

AM thanks CF and NSF grant AST-0098612 for support. RMR thanks NSF for grant AST-0709479.

\section{References}

Arnett, W. D. 1971, ApJ, 166, 153

Ballero, S. K., Matteucci, F. , Origlia, L., \& Rich, R. M. 2007, A\&A, 467, 123

Burris, D. L., Pilachowski, C. A., Armandroff, T. E., et al. 2000, ApJ, 544, 302

Cescutti, G., Matteucci, F., McWilliam, A., \& Chiappini, C. 2009, A\& $A$, 505, 605

Conti, P. S., Greenstein,J. L., Spinrad, H., Wallerstein, G., \& Vardya, M. S. 1967, ApJ, 148, 105

Fulbright, J. F., McWilliam, A., \& Rich, A. 2007, ApJ, 661, 1152

Lecureur, A., Hill, V., \& Zoccali, M., et al. 2007, A\&\&A, 465, 799

Maeder, A. 1992, A\&SA, 264, 105

Matteucci, F. \& Brocato, E. 1990, ApJ, 365, 539

McWilliam, A. \& Rich, R. M. 1994, ApJS, 91, 749

McWilliam, A. \& Rich, R. M. 2004, Origin and Evolution of the Elements, from the Carnegie Observatories Centennial Symposia. Carnegie Observatories Astrophysics Series. Edited by A. McWilliam and M. Rauch, 2004. Pasadena (arXiv:astro-ph/0312628)

McWilliam, A., Matteucci, F., Ballero, A., et al. 2008, AJ, 136, 367

Meynet, G. \& Maeder, A. 2002, A\&SA, 390, 561

Meléndez, J., Asplund, M. \& Alves-Brito, A., et al. 2008, A\&A, 484, L21

Nomoto, K., Thielemann, F-K., \& Yokoi, K. 1994, ApJ, 286, 644

Patsis, P. A., Skokos, Ch., \& Athanassoula, E. 2002, MNRAS, 337, 578

Smecker-Hane, T. \& Wyse, R. F. G. 1992, AJ, 103, 1621

Tinsley, B. M. 1979, ApJ, 229, 1046

Wallerstein, G. 1962, ApJS, 6, 407

Woosley, S. E. \& Weaver, T. A. 1995, ApJS, 101, 181

Wyse, R. F. G. \& Gilmore, G. 1993, ASP Conference Series, 48, 727

Zoccali, M., Hill, V., Lecureur, A., Barbuy, B., Renzini, A., et al. 2008, A\&AA, 486, 177 